\title{
The Reflection of Spherical Waves from Transition Zones with Arbitrary Depth-dependent Elastic Moduli and Density ${ }^{11}$
}

\author{
By \\ Karl Fuchs \\ Geophysical Institute, Universität Fridericiana, Karlsruhe, Germany
}

\begin{abstract}
Summary
A procedure has been presented for the numerical computation of synthetic seismograms of the reflected wave (including head waves and continuously refracted waves) arising from the incidence of a spherical wave on an inhomogeneous transition zone with arbitrary depth-dependent variation of the elastic moduli and density. The reflection phenomenon will be discussed in the frequency domain where the method of stationary phase gives a rapid estimate of the important features of the reflected wave field as a function of frequency, phase velocity and distance. Using this estimate synthetic seismograms are obtained by numerical integration with respect to wave number and by applying a fast Fourier transform technique. Synthetic seismogram sections for three typical examples of crust-mantle transition zones are discussed in detail. The procedure described in this paper provides a new means of placing more severe bounds on possible crustal and upper mantle models.
\end{abstract}

\section{§1. Introduction}

The knowledge of the depth-velocity distribution within the earth's interior, especially within the earth's crust and upper mantle has progressed considerably during the past 10 years. Until recently the crust, for instance, was believed to consist essentially of three homogeneous layers separated by more or less first-order discontinuities. Observation techniques have been improved both in quantity and quality. The interpretation of these new data resulted in rather complicated crustal models: the layers increased in numbers; homogeneous layers had to be replaced by layers with variable depthvelocity functions, transition zones replaced first-order discontinuities.

Classical methods for the inversion of $(T, A)$-data into depth-velocity models, for instance the Wiechert-Herglotz method, are

1) Contribution No. 109 within the research program "Determination of Crustal Structure in Central Europe" of the Deutsche Forschungsgemeinschaft (German Research Association). Contribution No. 32, Geophysical Institute, University of Karlsruhe. based on the validity of ray optics which implies that the change of the elastic moduli and density within one wavelength is negligible. This assumption is no longer valid in many recently deduced crustal models. Very often the wavelength is of the order of the layer thickness.

This is the present dilemma of most seismic travel time investigations of the crust: the derived models violate the very assumptions on which their derivation has been based. The same dilemma starts now for the upper mantle as high-quality data become available. What is needed at this stage is a precise knowledge of the wave propagation in arbitrary, inhomogeneous media. At present we do not know, how the seismograms for the derived crustal and upper mantle models would really look like. We do not even know whether the correlated phases can be used in estimating crustal and upper mantle models by classical inversion methods.

The exact solution for the equation of motion in inhomogeneous media has been obtained for very few cases only (Hook, 1961, 1962a, b, 1965; Scholte, 1961; Grant and 
West, 1965; Gupta, 1966). Even in the case of vertical incidence upon a transversely homogeneous medium with the main parameters changing only in the vertical direction, an analytical solution has been found for special cases only (Sezawa and Kanai, 1935; Wolf, 1937; Menzel and Rosenbach, 1957, 1958a, b; Bortfeld, 1960; Datta, 1967).

Since seismic signals are band limited an approximate solution for the problem of wave propagation in inhomogeneous media, obtained within the frequency band of the incident signal, is sufficient for most purposes. By dividing an inhomogeneous medium into a series of sufficiently thin homogeneous layers it is possible to simulate wave propagation in an inhomogeneous layer within a given frequency range to any desired accuracy.

For waves from point sources two methods have been used so far, which may be characterized under the heading of generalized ray theory (Pekeris, 1955a, b, 1960; Pekeris and Lifson, 1957; Spencer, 1965, 1965; Bortfeld, 1962a, b, 1964, 1967; Pekeris, Alterman and Abramovici, 1963; G. Müller, 1964, 1967; Pekeris, Alterman, Abramovici and Jarosch, 1965; Helmberger, 1968) and wave theory in layered media (Thomson, 1950; Haskell, 1953; Harkrider, 1964; Phinney, 1965). In the first method all possible multiple reflections are summed up in the time domain. If the layers become very thin, the problem arises where the infinite series of multiple reflections should be truncated.-The second method is applied in the frequency domain using the Thomson-Haskell matrix formalism. The solution includes automatically all multiple reflections. Sufficient frequency resolution for models with long time responses might cause storage and computing time problems discussed in detail by Phinney (1965).

In this paper the numerical computation of reflections arising from the incidence of spherical wave on an inhomogeneous transition zone will be carried out in the frequency domain where the method of stationary phase permits to give a rapid estimate of the pattern of the reflected wave field as a function of frequency, phase velocity and distance.-Synthetic seismograms are obtained by numerical integration with respect to wave number and fast inverse Fourier transformation (Cooley and Tukey, 1965). The comparison of observed and synthesized seismograms will provide a new means of placing more severe bounds on possible crustal and upper mantle models.

\section{$\S 2$. The Reflection of Spherical Waves from Transition Zones}

In studying reflected and refracted body waves from inhomogeneous transition zones within crustal and upper mantle models at some depth the following model is used throughout this paper (Fig. 1). A layered transition zone is placed between two homogeneous half spaces at depth $h$ below a point source $S$ located in the upper half space. The receiver $E$ is at horizontal distance $r$ and at depth $z$ below the source. There is no free surface in this model. The omission of waves multiply reflected between the free surface and the transition zone corresponds to a partial ray expansion in the upper half space.

The displacement potential of the reflected compressional wave in the frequency domain arising from the incidence of a spherical compressional wave upon the layered transition zone is:

$$
\begin{aligned}
& \bar{\varphi}(r, z, \omega) \\
& \quad=L(\omega) \int_{0}^{\infty} \tilde{R}_{P P}(\omega, k) \cdot e^{+j \sigma_{0}(z-2 h)} J_{0}(k r) \frac{k d k}{j \sigma_{0}}
\end{aligned}
$$

$\widetilde{R_{P P}}$ is the complex reflectivity of the transition zone for plane waves of type PP. $L(\omega)$ is the Fourier transform of the displacement potential for the incident signal. $k$ and $\sigma_{0}$ are the horizontal and vertical wave numbers in the upper half space, respectively. $r$ is the horizontal distance between source and receiver.

If there is a stack of comparatively thick homogeneous layers on top of the transition zone partial ray expansion may be applied 


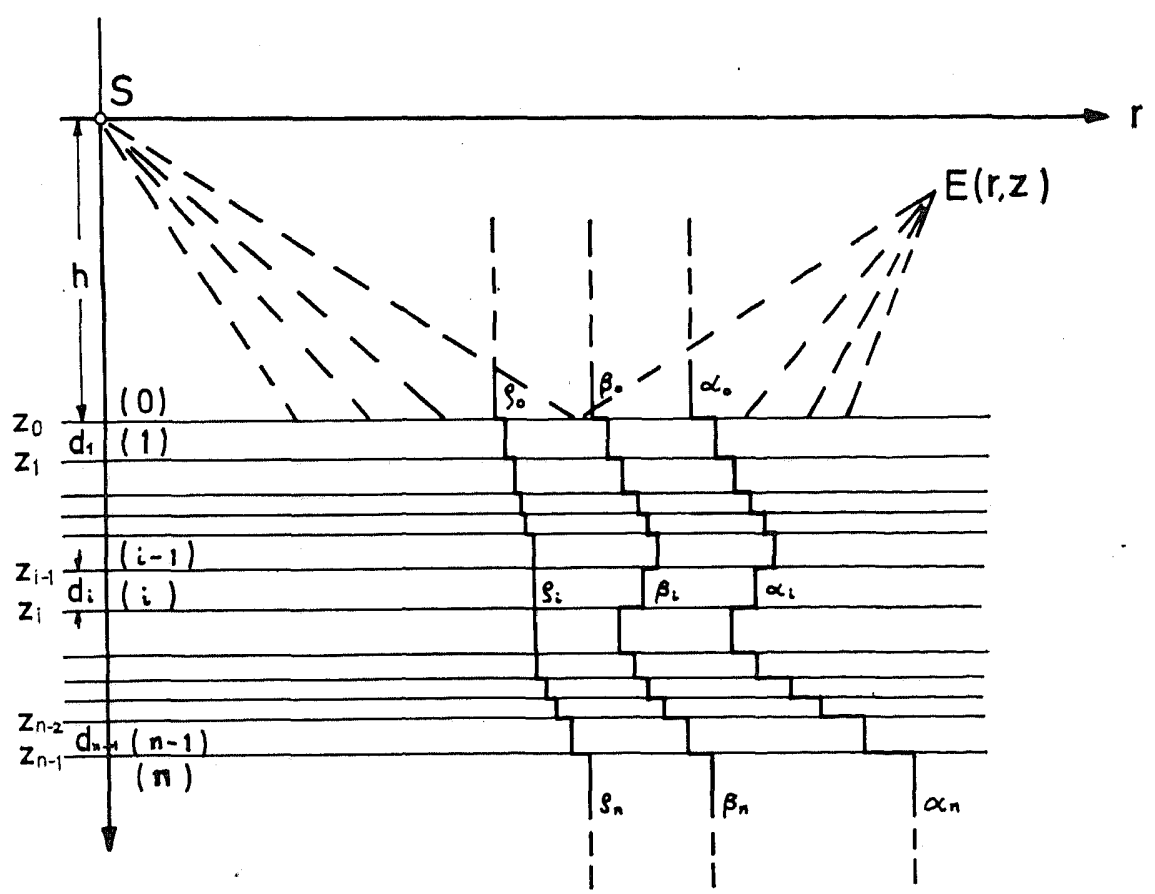

Fig. 1. Transition zone between two homogeneous half spaces. The arbitrary depth-dependent distribution of the elastic moduli and density is approximated by an arbitrary number of thin homogeneous layers with arbitrary $P$ - and $S$-wave velocity, density and thickness $\left\langle\alpha_{i}, \beta_{i}, \rho i\right.$ and $d_{i}$ respectively). Source $S$ and receiver $E$ are in the upper half space.

which again leads to similar expressions where only the exponent takes the form of a sum over the layers in the stack, and the integrand must be multiplied by the product of the corresponding transmission coefficients.

If $k$ is larger than the total wave number $k_{\alpha_{0}}$ in the upper half space the exponent in the integrand in eq. (1) becomes real and negative. If $(2 h-z)$ is sufficiently large the contributions for $k>k_{\alpha_{0}}$ will become negligible. Therefore we restrict the integration over $k$ to the interval $\left\langle 0, k_{\alpha_{0}}\right\rangle$. This corresponds to integration over real angles of incidence from $0^{\circ}$ to $90^{\circ}$. By this restriction all interface waves with phase velocities smaller than the $P$-velocity in the upper half space are excluded from the solution. In other words, either the source must be located sufficiently far away from the transition zone so that interface waves of the Stoneleytype are not generated to any sizable degree, or if they are generated the receiver has to be at such a large distance from the transition zone that the amplitude of these transversely damped waves have decayed sufficiently.

If $\gamma$ is the angle of incidence at the top of the transition zone, the vertical displacement of the reflected wave in the upper half space is derived from eq. (1) by substituting $k=k_{\alpha_{0}} \cdot \sin \gamma$ :

$$
\begin{aligned}
\bar{w}_{0}(z, r, \omega)= & \frac{\partial \vec{\varphi}}{\partial z} \\
= & L(\omega) \int_{0}^{90^{\circ}} \tilde{R}_{P P}(\omega, \gamma) e^{j k_{\alpha 0} \cdot \cos \gamma(z-2 h)} \\
& \times J_{0}\left(r k_{\alpha_{0}} \sin \gamma\right) k_{\alpha_{0}}^{2} \sin r \cos \gamma d \gamma
\end{aligned}
$$

The assumptions used throughout this paper may be summarized as follows:

- The free surface is omitted. Therefore, no surface waves have to be considered, and there is no interference with multiple reflections from the free surface.

$-(2 h-z)$ must be sufficiently large to 


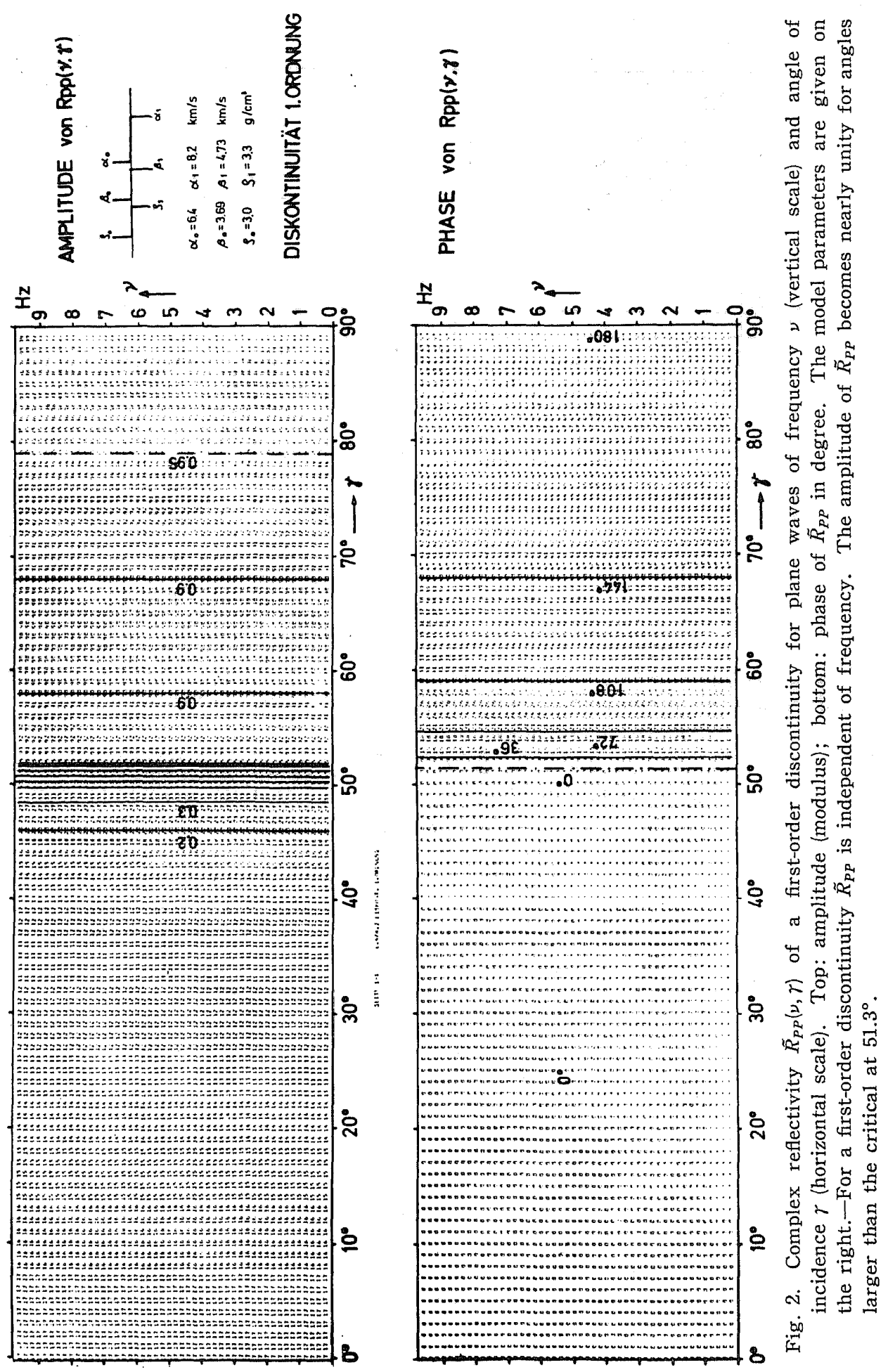




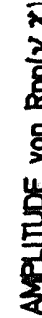


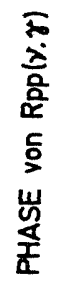

농.․․ㄴㅇㅇ
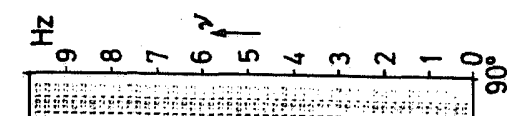

N.
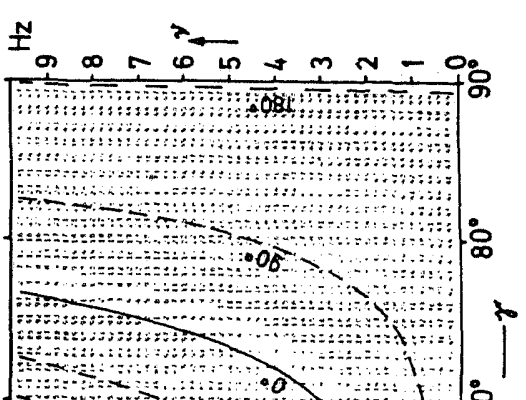

छี

茪岁泀

焉

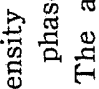

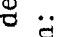

突苞

象



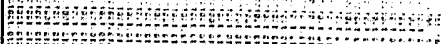
Fon E.

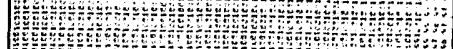
mons Ton 20
3

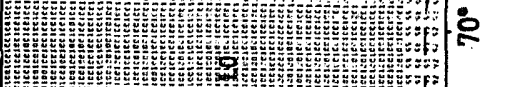

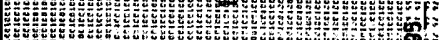

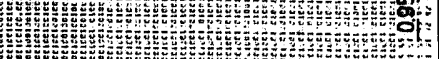

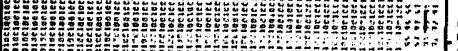

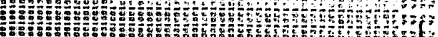
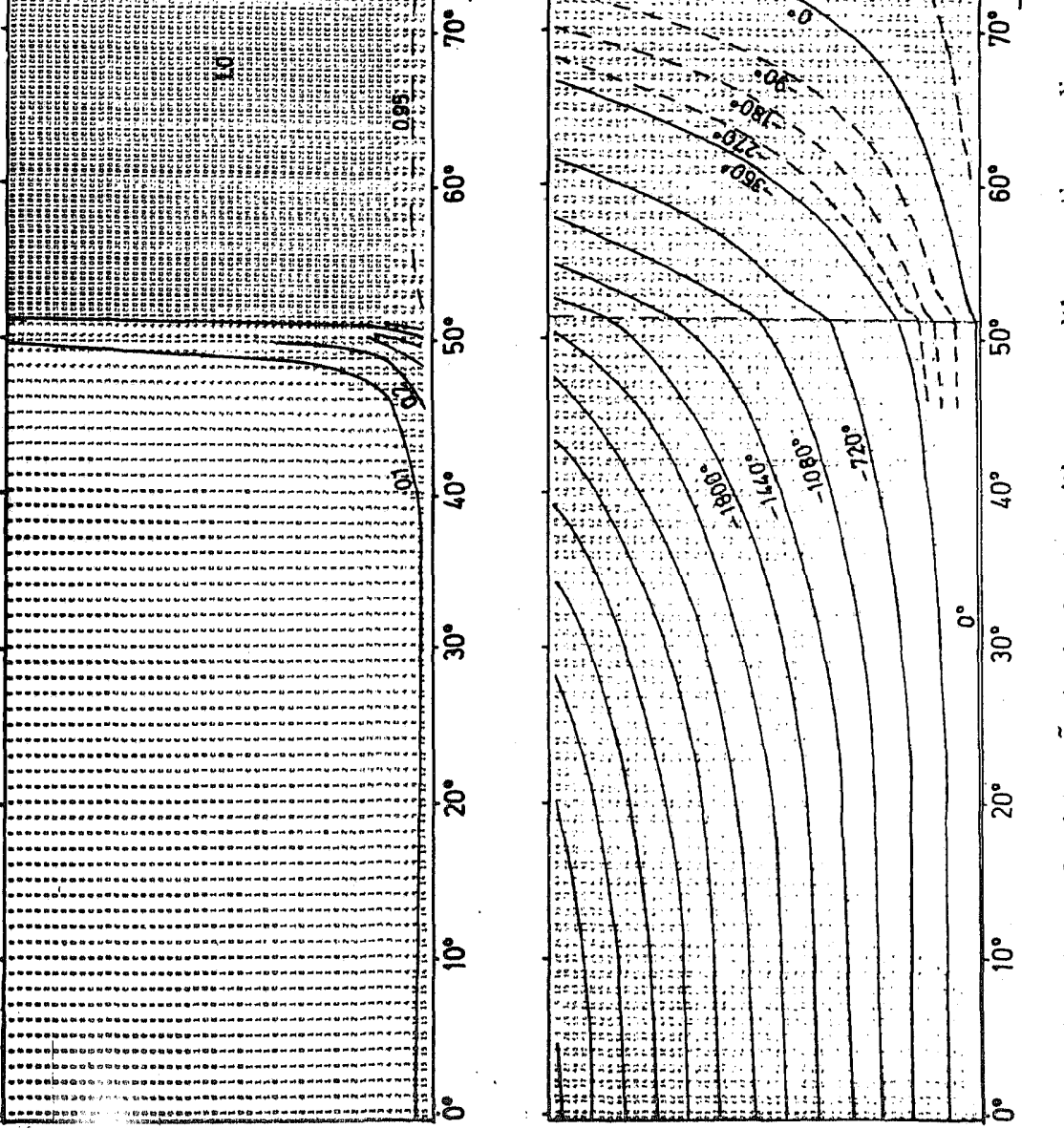

궁

Ð ⿷匚⿱乛⿻上丨

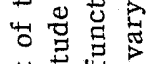

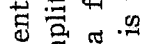



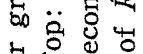

कैं

青完量

苋骂导

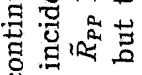

出 है

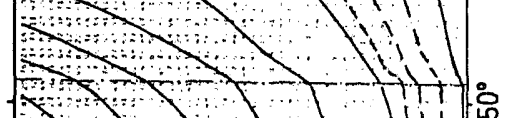

专

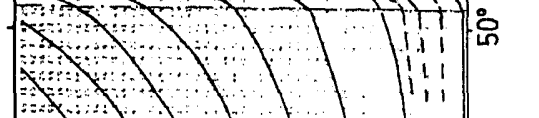

ह 进范

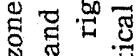

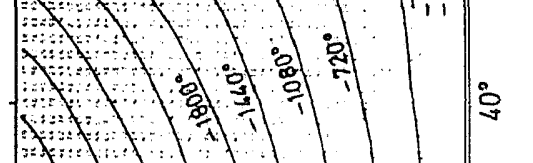

I 2 焉

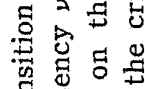

劳要

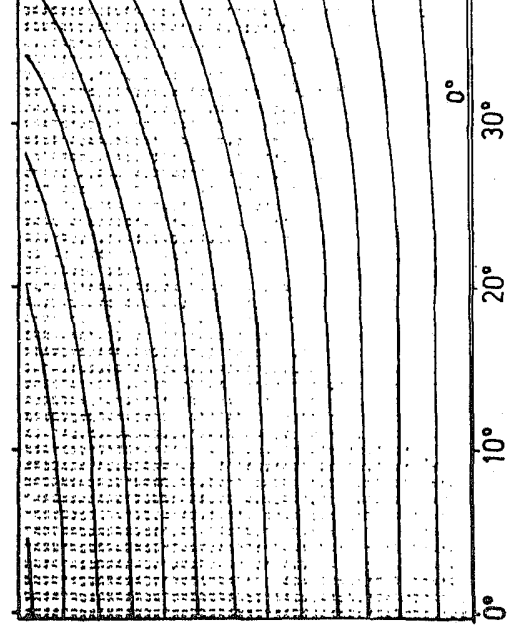

( $)$

岁岁矛点

뇽

중ㄹ⿺

स्ञ

忩䍃总

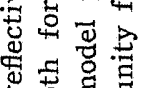

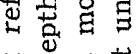

ه

药

ठำ

क 范范

$\ddot{0}$ 
exclude interface waves of the Stoneley type, so that integration over only real angles of incidence is justified.

- The interfaces are plane and horizontal. Although these assumptions restrict the general applicability of the present solution, body wave phases considered in the investigation of the deeper crust and upper mantle will not be affected by these assumptions. Two methods of evaluating the integral will be reported in this paper: (1) the method of the stationary phase, (2) direct numerical integration over real angles of incidence.

\subsection{The method of stationary phase}

The method of stationary phase is applied to obtain an approximate solution which is very useful in rapidly estimating the pattern of the reflected wave field as a function of distance and phase velocity. This method is discussed in detail by Fuchs $(1969 \mathrm{a}, \mathrm{b})$. Therefore only the main results will be presented here.

The complex reflectivity $\tilde{R}_{P P}$ is a comparatively slowly varying function of the angle of incidence, especially for overcritical incidence. To be more precise, this is true for the amplitude (modulus) of the complex reflectivity $\tilde{R}_{P P}$ while the phase may change rapidly. The reflectivity of a first-order discontinuity (Fig. 2) is independent of frequency. For angles larger than the critical angle the amplitude of the reflectivity is approximately unity while the phase varies slowly from $0^{\circ}$ to $180^{\circ}$. Even for more complicated transition zones the amplitude of the reflectivity is very close to unity beyond the critical angle. In Fig. 3 the reflectivity of a transition zone with a continuous linear gradient between 25 and $30 \mathrm{~km}$ depth is depicted. The rapid variation of the phase is in contrast to the constant amplitude for overcritical angles of incidence.

For this reasons we do not consider the complex quantity $\widetilde{R}_{P P}$ as a slowly varying function of $\gamma$ but only its amplitude. The phase $\phi$ of $\tilde{R}_{P P}$ is to be included in the determination of the stationary phase of the integrand in eq. (2). Following Brekhovskikh
(1960) it is useful to introduce the ray displacement $\Delta$ :

$$
\Delta\left(\omega, \gamma_{0}\right)=\left(\frac{\partial \phi / \partial \gamma}{k_{\alpha_{0}} \cos \gamma}\right)_{\gamma=\gamma_{0}} .
$$

At a distance $r$ the stationary phase $\gamma=\gamma_{0}$ is a solution of the equation:

$$
r=2 h \cdot \operatorname{tg} \gamma_{0}+\Delta\left(\nu, \gamma_{0}\right) \quad \text { with } \quad \nu=\frac{\omega}{2 \pi} .
$$

Since the ray displacement $\Delta$ in the transition zone is a function of frequency $\nu$, the stationary phase becomes a function of distance $r$, depth $h$ of the transition zone and frequency. The concept of ray displacement is illustrated in the lower left of Fig. 4. The formula for the Fourier transformed total displacement $\bar{u}(r, \omega)$ for the stationary phase $\gamma_{0}$ is also given in Fig 4.

For a first-order discontinuity one finds two solutions $\gamma_{01}$ and $\gamma_{02}$ of eq. (4) for angles of incidence larger than the critical angle $\gamma_{c r}$. The stationary phase $\gamma_{02}$ close to the critical angle corresponds to the head wave guided in the lower half space. The other solution $\gamma_{01}$ corresponds to the overcritical reflection from the first-order discontinuity. Graphical representations of

$$
2 h \cdot \operatorname{tg} \gamma_{0}+\Delta\left(\nu, \gamma_{0}\right)=\text { const. }
$$

are given on the right in Fig. 4 for the case of a first-order discontinuity at a depth of $30 \mathrm{~km}$ for frequencies $0.2,0.6,1.0,3,5,7$ and $9 \mathrm{~Hz}$ at angles larger than critical. From these curves the location of the stationary phases may be determined as a function of distance $r$ and frequency $\nu$. In the high-frequency limit the stationary phases are approaching the angle of incidence according to ray theory. The ray displacement $\Delta$ is going to zero for the branch $\gamma_{01}$ of the overcritical reflection, while the head wave branch $\gamma_{02}$ is approaching a constant, namely the critical angle $\gamma_{c r}$. For $\gamma_{02}$ the ray displacement comes close to $r-r_{\text {crit }}\left(r_{\text {crit }}\right.$ $=$ critical distance), that is the distance a wave travelled horizontally along the interface with the $P$-velocity of the lower half space.

For finite frequencies the two branches of 
REFLECTED SPHERICAL WAVE WITH RAY DISPLACEMENT

$$
\begin{aligned}
& \bar{u}(r, \omega)=\tilde{L}(\omega) \cdot \frac{R_{P P} e^{-j k_{\alpha}\left(\sin \gamma_{0} \Delta\left(\omega, \gamma_{0}\right)+S_{0}\right)}}{\sqrt{\left(S_{0}+\frac{\Delta\left(\omega, \gamma_{0}\right)}{\sin \gamma_{0}}\right)\left(\left|S_{0}+\sin \gamma_{0} \Delta+\frac{\phi^{\prime \prime}\left(\omega, \gamma_{0}\right)}{k_{\alpha}}\right|\right)}} \\
& \Delta\left(\omega, \gamma_{0}\right)=\left(\frac{\partial \phi / \partial \gamma}{k_{\alpha} \cos \gamma}\right)_{\gamma=\gamma_{0}} \text { RAY DISPLACEMENT } \\
& \text { S. RAY PATH IN UPPER HALF SPACE } \\
& \phi(\omega, \gamma) \text { PHASE OF REFLECTIVITY R } \tilde{R}_{\text {PP }} \\
& \phi^{\prime \prime}\left(\omega, \gamma_{0}\right)=\left(\frac{\partial^{2} \phi^{2}}{\partial \gamma^{2}}\right)_{\gamma \cdot \gamma_{0}}
\end{aligned}
$$



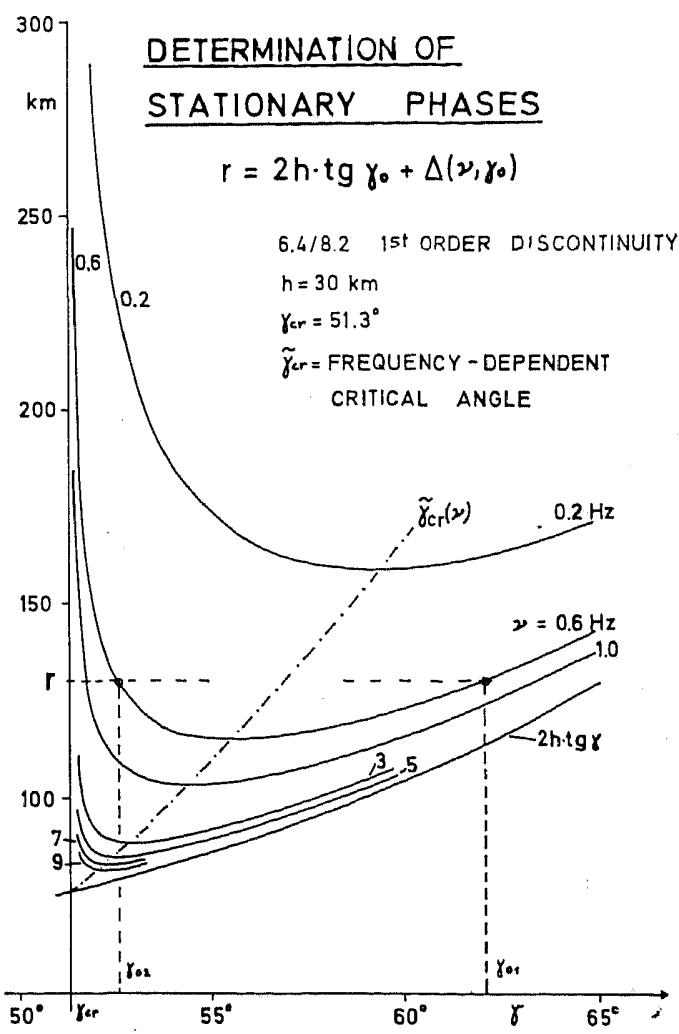

Fig. 4. Determination of stationary phases. - The stationary phases $\gamma_{0}$ are solutions of eq. (4). Upper left: contribution of the stationary phase $\gamma_{0}$ to the total displacement in the frequency domain. $\tilde{L}(\omega)$ is the Fourier transformed total displacement of the incident signal in the far field. - Lower left: illustration of the concept of ray displacement. There may be several solutions for a fixed distance and frequency. -Right: locations of stationary phases of a first-order discontinuity for overcritical angles of incidence.

$\gamma_{01}$ and $\gamma_{02}$ join each other at an angle $\tilde{\gamma}_{o r}$ which is larger than the critical angle $\gamma_{o r}$ from ray theory. This frequency-dependent critical angle $\tilde{\gamma}_{c r}(\nu)$ is larger for smaller frequencies. The corresponding frequency-dependent critical distance is shifted to larger distances. This result is in good agreement with earlier statements by Červený (1961).

Figure 5 gives the comparison of the stationary phases for a first-order discontinuity at a depth of $30 \mathrm{~km}$ (left hand side) and a transition zone (right hand side of Fig. 5) with a continuous linear increase of the velocities and density between 25 and $30 \mathrm{~km}$. The model parameters are the same as in Figs. 2 and 3, respectively. The ray displacement is considerably larger for the transition zone than for the first-order discontinuity. In this figure the angle of incidence has also been expressed in horizontal phase velocity $c$ :

$$
c=\alpha_{0} / \sin \gamma
$$

as indicated on the upper scale. Attention should be drawn to the normal dispersion $(\partial c / \partial \nu>0)$ of the "head wave" and to the anomalous dispersion $(\partial c / \partial \nu<0)$ of the "overcritical reflection."

Computation of synthetic seismograms by summation of the contribution from the 

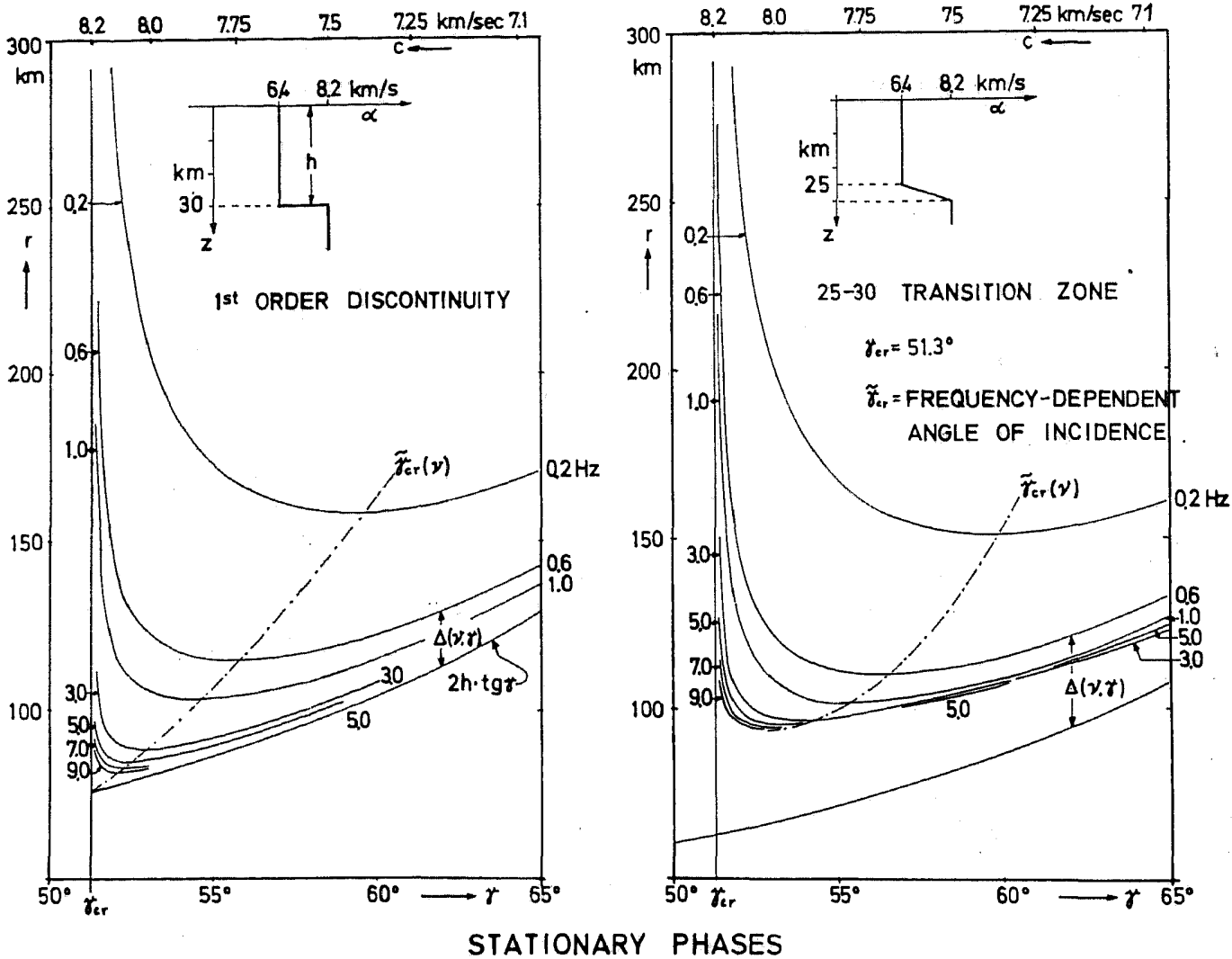

Fig. 5. Comparison of stationary phases for a first-order discontinuity at a depth of $30 \mathrm{~km}$ and a transition zone with a continuous linear gradient of the elastic moduli and density between 25 and $30 \mathrm{~km}$. For model parameters compare Figs. 2 and 3, respectively. Note the larger ray displacement in case of the transition zone.

stationary phases at distance $r$ followed by a fast inverse Fourier transformation has been attempted unsuccessfully. It has finally been abandoned for two reasons:

(1) the contribution of the stationary phase could only be determined by numerically locating the stationary phase and then computing the first and second derivative $\phi^{\prime}$ and $\phi^{\prime \prime}$ of the phase $\phi$ of $\tilde{R}_{P P}$ with respect to frequency. Especially $\phi^{\prime \prime}$ was contaminated by numerical noise;

(2) for more complicated models (see Fig. 6) there may be more than two stationary phases for each frequency at one particular distance. In this case the search-time on the computer may become larger than the time for direct numerical integration of eq. (2).

Two examples of a transition zone (linear gradient zone below a first-order discontinuity) with multiple stationary phases in a frequency band used in crustal and upper mantle investigations are given in Fig. 6 . As is seen the multiple stationary phases between the branches of the head wave and the overcritical reflection extend only to finite distances. It will be shown in the next section that this finite range is also observed in synthetic seismogram sections obtained by direct numerical integration. The range of the multiple stationary phases 


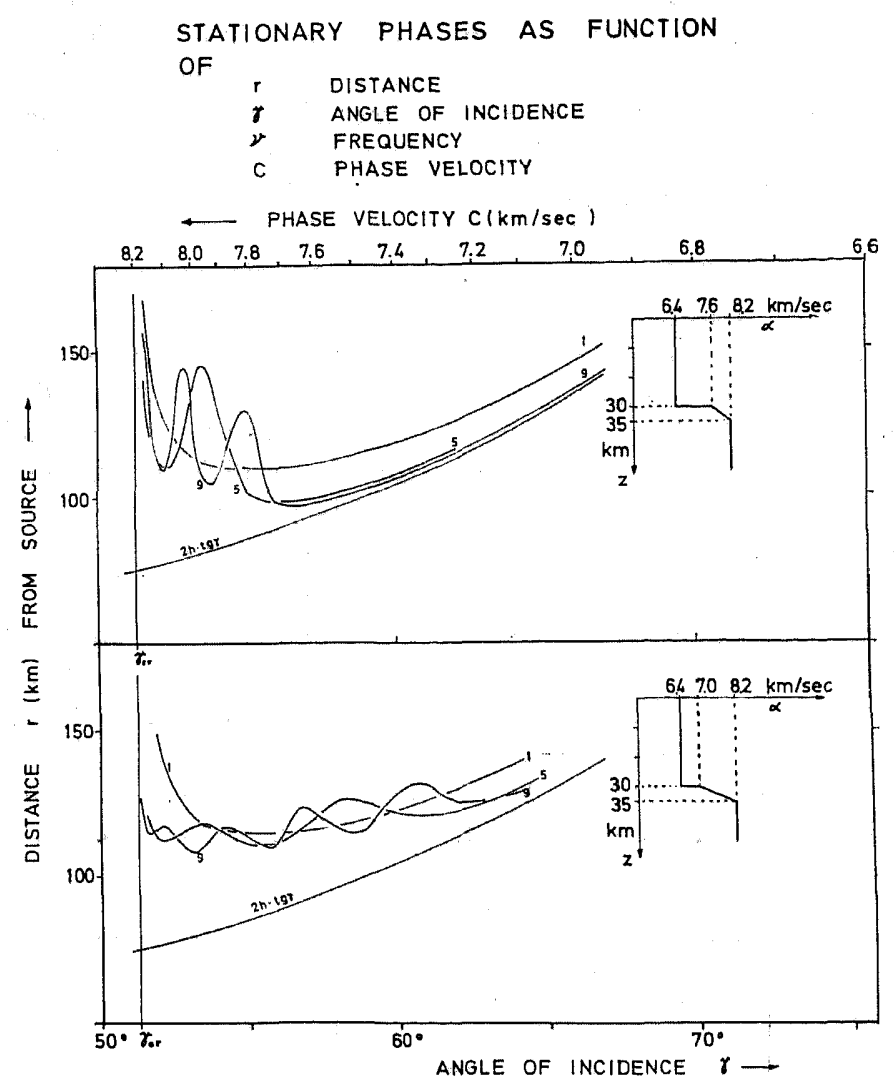

Fig. 6. Multiple stationary phase in case of linear gradient zones below a first-order discontinuity. The $P$-wave velocities are given in the Figure. The corresponding $S$-wave velocities and densities are: top-3.69, 4.38. $4.73 \mathrm{~km} / \mathrm{s} ; 3.0,3.2,3.3 \mathrm{~g} / \mathrm{cm}^{3} ;$ bottom-3.69, $4.04,4.73 \mathrm{~km} / \mathrm{s} ; 3.0,3.1$, $3.3 \mathrm{~g} / \mathrm{cm}^{3}$. Note the finite distance range of the multiple stationary phases. Synthetic seismograms for the top model are given in Fig. 9 .

depends upon the gradient and the thickness of the transition zone. Their phase velocity lie in the range of $P$-velocities encountered in the transition zone.

Although computation of synthetic seismograms by the summation of contributions from all stationary phases was not feasible, the calculation of the location of the stationary phases for selected frequencies proved to be a very fast and effectiva way to estimate the main features of the reflected wave field. This will be demonstrated in the next section where synthetic seismograms are obtained direct numerical integration.

\subsection{Synthetic seismograms by direct numeri- cal integration}

Synthetic seismograms for the vertical displacement of the waves reflected from transition zone with arbitrary depth dependent distribution of the elastic moduli and density are computed in this section from eq. (2). The integral in this expression is evaluated numerically. The large argument approximation for the Bessel function $\mathrm{J}_{0}$ is used for distances larger than $30 \mathrm{~km}$. This is well justified considering the wave numbers occurring in refraction studies of the crust and upper mantle. Inverse Fourier 
transformation from the frequency into the time domain is performed by a fast computing technique (Cooley and Tukey, 1965).

In a first step the matrix of the complex reflectivity $\tilde{R}_{P P}$ is computed as a function of frequency and angle of incidence. An angle increment of $0.25^{\circ}$ has been found to be sufficient in the cases studied so far. The $\tilde{R}_{P P}$ are then stored on magnetic disk and are ready for computation of a synthetic seismogram at any desired distance. Especially for complicated models the computation of $\tilde{R}_{P P}$ requires most of the computer time (example: a 12 layer model with 360 angles and 1000 frequencies takes about 30 minutes on the IBM 7094). Once the $\tilde{R}_{P P}$ are available from storage integration over the angles of incidence together with a fast inverse Fourier transformation is performed within 1-3 minutes for each distance r.-Especially at large distances the phase of the integrand is varying rapidly. But the main part of this variation corresponds only to the travel time and does not affect the shape of the reflected signal. If $t_{0}$ is the travel time of the fastest traveling phase arriving at a certain distance the contribution of the travel time to the phase may be removed by adding $\omega t_{0}$ to the phase. In the time domain this is equivalent to shifting the seismogram by $t_{0}$ to earlier times. In the following synthetic seismogram sections all times have been reduced with $t_{0}=r / 8.2 \mathrm{sec}$.

Examples of synthetic seismogram sections for three different models will be discussed in this paper:

- first-order discontinuity (Fig. 7)

- linear gradient zone (Fig. 8)

- linear gradient zone below a firstorder discontinuity (Fig. 9).

The model parameters $(P$ - and $S$-wave velocities as well as densities) as a function of depth are depicted in the lower left of Figs. 7-9. Also given in the figures are the

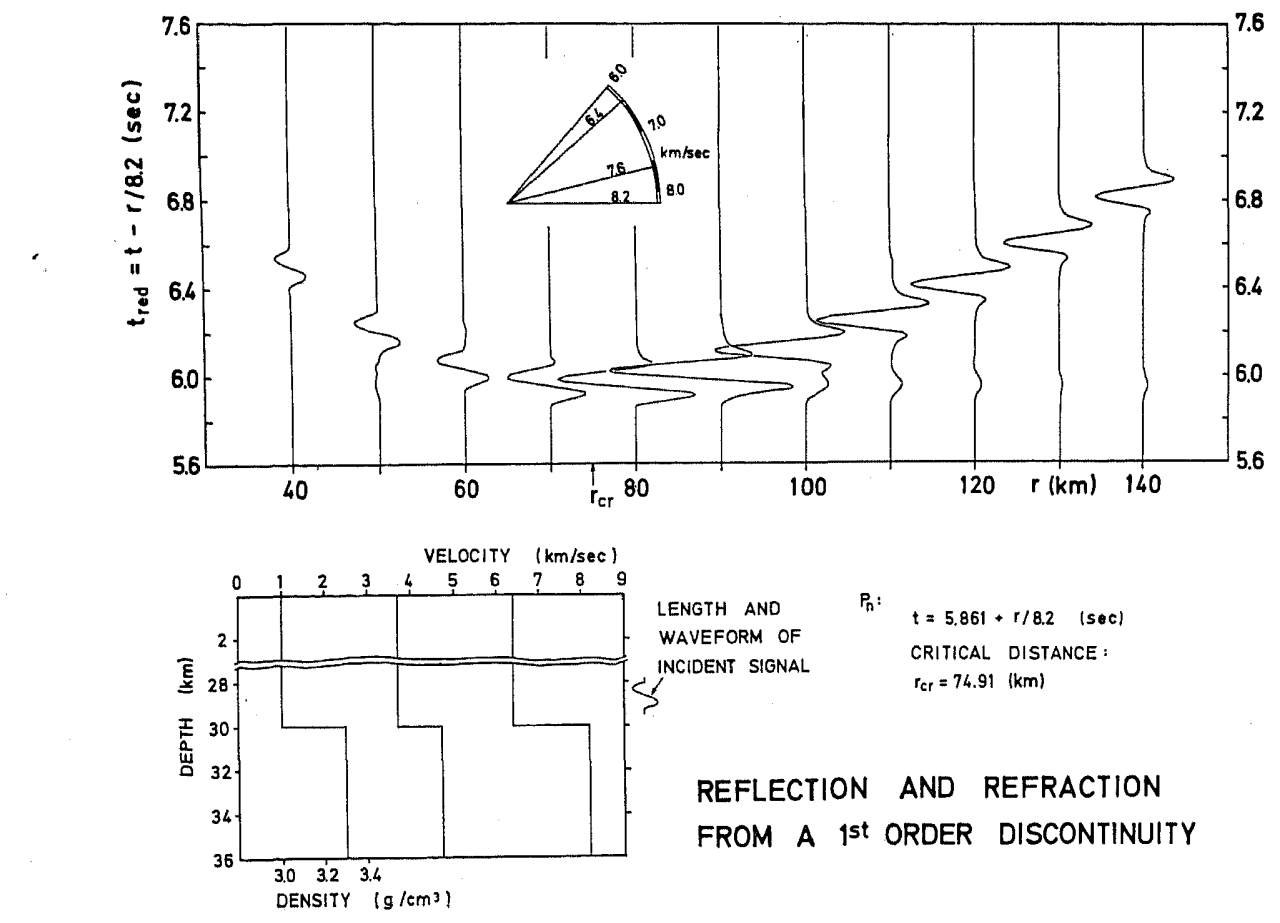

Fig. 7. Section of synthetic seismograms from a first order discontinuity at a depth of $30 \mathrm{~km}$. Times are reduced with $r / 8.2 \mathrm{sec}$. 


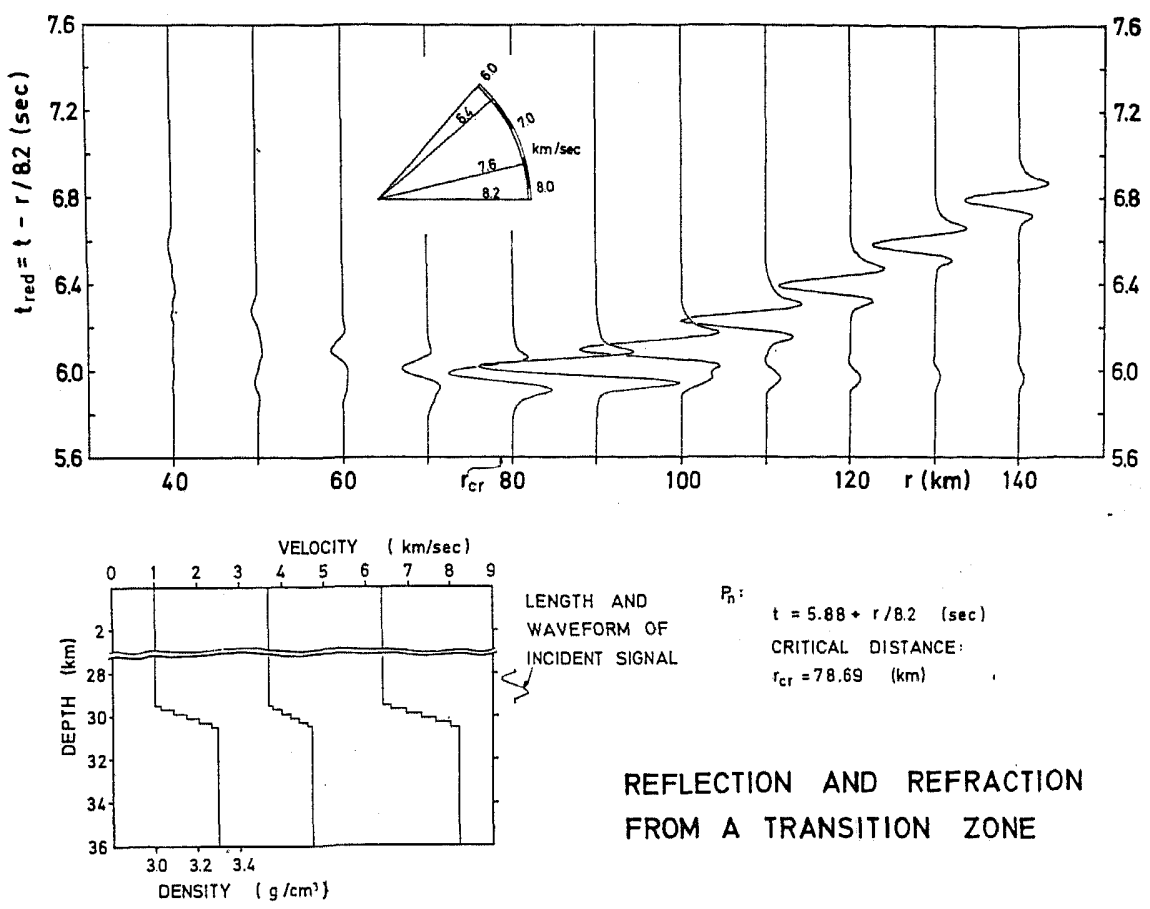

Fig. 8. Section of synthetic seismograms from a transition zone at a depth between 29.5 and $30.5 \mathrm{~km}$ where the velocities and density increase linearly with depth in 5 small steps. Times are reduced with $r / 8.2 \mathrm{sec}$. Note the vanishing amplitude for distances smaller than critical and the sharper onset of the overcritical reflection which is now a signal continuously refracted out of the transition zone.

critical distances for $P_{n}$ and $P_{i}$ and the length and waveform of the incident signal scaled to the depth axis of the models. The incident signal is:

$$
f(t)=\left\{\begin{aligned}
& \sin 10 \pi t-0.5 \sin 20 \pi t \\
& \text { for } 0 \leq t \leq 0.2 \mathrm{sec} \\
& 0 \text { for } t<0 \text { and } t>0.2 \mathrm{sec}
\end{aligned}\right.
$$

The synthetic seismograms from a firstorder discontinuity in Fig. 7 serve as a test of the accuracy of the method. The following points should be noted: The $P_{n}$ head wave has the form of the time integral of the incident signal in agreement with the theory of head waves (e.g. Heelan, 1953). -The maximum amplitudes do not occur at the critical distance $r_{o r}$ but are displaced to larger distances. This is concordant with the results of Červený (1961).-The overcritical reflection shows dispersion undergoing a phase change of nearly $180^{\circ}$ between the critical distance and $140 \mathrm{~km}$.

In Fig. 8 the first-order discontinuity has been replaced by a transition zone of $1 \mathrm{~km}$ thickness where the velocities and density are increasing linearly with depth in 5 small steps. Within the frequency band of the incident signal this transition zone forms a good approximation to a linear and continuous increase with depth. The main differences compared to the reflections from a first-order discontinuity are as follows:

The amplitudes of the reflected signal at distances less than the critical distance are very small.-No multiple reflections and head waves from the intermediate layers are to be observed in the case of the incident signal used in the present calculations.- 

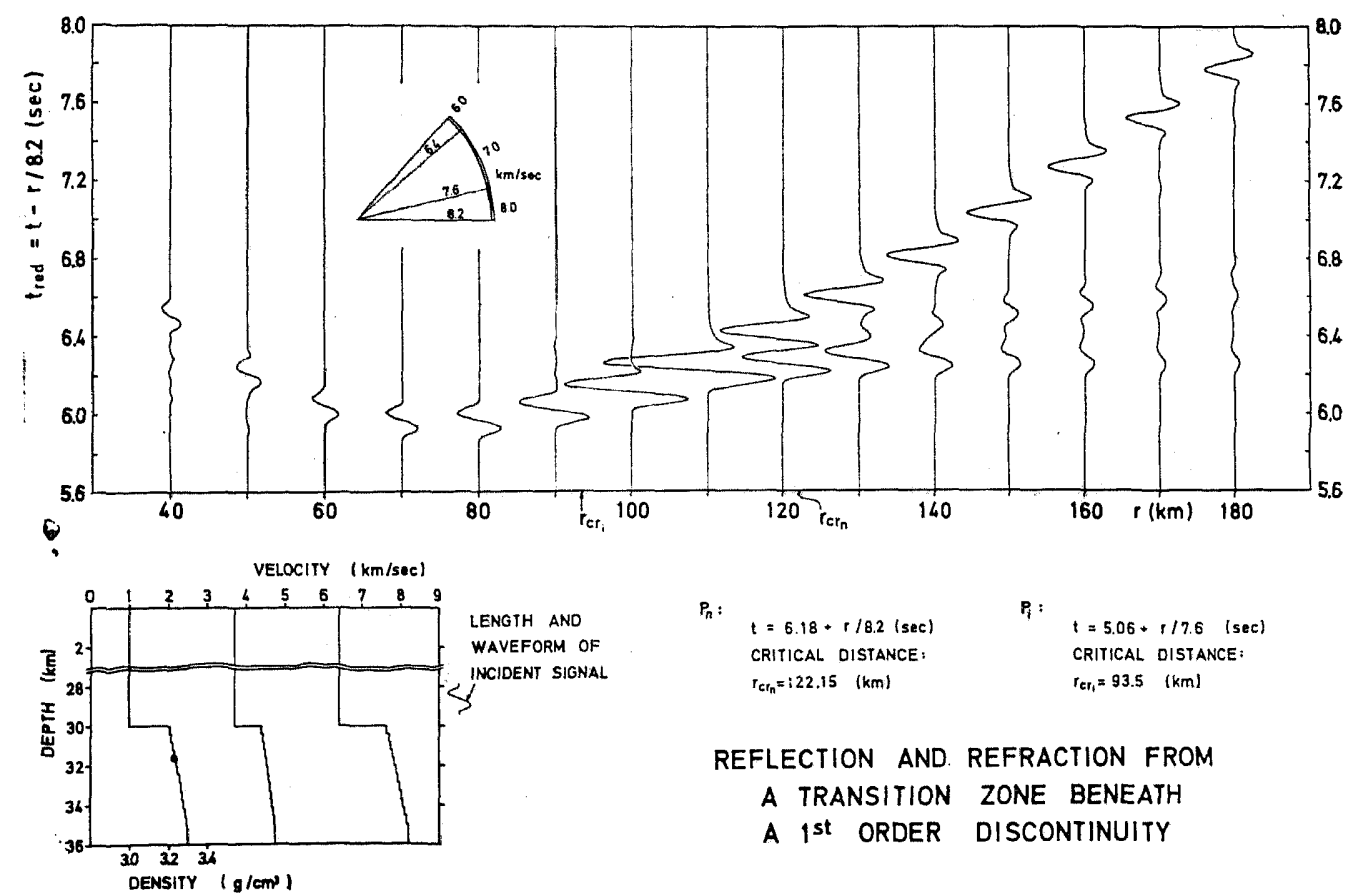

$P_{n}$ :

$t=6.18+r / 8.2(\mathrm{sec})$ CRITICAL DISTANCE: $r_{C_{\mathrm{n}}}=22.15(\mathrm{~km})$

$P$ : $t=5.06 * r / 7.6 \quad|\mathrm{sec}|$ CRITICAL DISTANCE; $r_{C r}=93.5 \mathrm{kmm}$

\section{REFLECTION AND. REFRACTION FROM \\ A TRANSITION ZONE BENEATH \\ A Ist ORDER DISCONTINUITY}

Fig. 9. Section of synthetic seismograms from a transition zone of $5 \mathrm{~km}$ thickness below a first-order discontinuity at a depth of $30 \mathrm{~km}$. In the transition zone the velocities and density increase linearly with depth in 12 small steps and merge continuously into the lower half space. The reverberations following the $P_{n}$-signal are the multiple stationary phases (compare top of Fig. 6). They form the system of multiple reflections being continuousiy refracted out of the transition zone and interfering constructively with each other. Times are reduced with $r / 8.2$ sec.

Here a continuously refracted wave takes the place of the overcritical reflection. It differs from the reflection signal by a sharper onset. -The maximum amplitudes are again displaced to large distances.

Before discussing the synthetic seismograms for a gradient zone below a first-order discontinuity in Fig. 9, the position of the multiple stationary phases computed for the same model should be recalled (Fig. 6, top). They are extending to a distance of about $150 \mathrm{~km}$ with phase velocities slighty smaller than the velocity of the head wave in the lower half space. Computation of synthetic seismograms for this model will answer a number of questions: How do the multiple stationary phases appear in the time domain? What is the amplitude of these stationary phases compared to the reflection and head wave amplitudes? Is the range of the multiple stationary phases the same as that estimated from the location of the multiple stationary phases in the frequency domain?

The synthetic seismogram section for this model of a gradient zone below a first-order discontinuity is given in Fig. 9. Special attention should be paid to the following observations: The $P_{n}$ phase, i.e. the onset of the head wave travelling in the lower half space is not tangent to the hyperbola of the reflections from the first-order discontinuity.

The secondary events following the $P_{n}$ phase cannot be explained by simple geometrical ray theory. They form the system of multiple reflections being continuously refracted out of the transition zone and inter- 
fering constructively with each other. This system of constructively interfering multiple reflections is propagating within the transition zone. These are the multiple stationary phases discussed in the previous section (Fig. 5). Their phase velocity ranges from 7.6 to $8.2 \mathrm{~km} / \mathrm{s}$. This range depends on the spectrum of the incident signal. In the limit of a very strong gradient this wave group gradually merges into another head wave guided at the $6.4 / 7.6 \mathrm{~km} / \mathrm{s}$ discontinuity. Reverberations of the $P_{n}$ signal of such a type are very often observed in crustal refraction studies.

The amplitudes of the secondary events are strongly decreasing beyond a distance of $160 \mathrm{~km}$. This is in good agreement with the distance range of the multiple stationary phases discussed above.-The attempt to interpret these secondary events by methods derived from classical ray theory results in false depth-velocity models. - The occurance of these multiple stationary phases seems to be a criterion for the sharpness of a transition zone.

Between a distance of 93.5 and $122.2 \mathrm{~km}$ the wave continuously refracted within the transition zone forms the first arrival. The large amplitudes of this phase are comparable to the amplitudes of the overcritical reflection. Its wave form is that of the incident signal. Only beyond the critical distance for $P_{n}$ the signal gradually takes the form of the time integral of the incident signal.

\section{§3. Conclusion}

A procedure for the rapid estimation of the main features of the reflected wave field arising from the incidence of a spherical wave upon a transition zone with arbitrary depth-dependent distribution of the elastic moduli and density has been developed by applying the method of stationary phase to the integral representation of the reflected wave. This estimate is then used to determine the distances at which synthetic seismograms should be computed. Within the synthetic seismograms obtained by the more accurate method of direct numerical integration the multiple stationary phases are associated with secondary events or reverberations of the head wave signal. The occurence of these secondary events is an indication of the sharpness of the transition zone.

The investigation of a number of problem has become feasible by the method described in this paper, e.g. wave propagation in laminated media, wave propagation in transversely anisotropic media. The method will be applied to the study of body wave propagation in inhomogeneous media. The comparison of observed and computed seismograms provides an effective means to extract more information from seismogram sections. This will put more severe bounds on crustal and upper mantle models.

\section{§ 4. Acknowledgements}

The author is indebted to his colleagues at the Geophysical Institute of Karlsruhe University, especially to Professor Stephan Mueller for many helpful discussions, his encouragement and for critically reading the manuscript. This study has been sponsored by the German Research Association (Deutsche Forschungsgemeinschaft). Computing facilities were made availabe by the German Computing Center (Deutsches Rechenzentrum) at Darmstadt.

\section{References}

BORTFELD, R.:

1960 Seismic waves in transition layers, Geophys. Prosp., 8, 2, 178-217.

BORTFELD, R.:

1962a Exact solution of the reflection and refraction of arbitrary spherical compressional waves at liquid-liquid interfaces and at solid-solid interfaces with equal shear velocities and equal densities, Geophys. Prosp., 10, 35-67.

BORTFELD, R.:

1962b Reflection and refraction of spherical compressional waves at arbitrary plane interfaces, Geophys, Prosp., 10, 517-538.

BORTFELD, R.:

1964 Examples of synthetic refraction arrivals and wide-angle reflections, Geophys. 
Prosp., 12, 1, 100-114.

BORTFELD, R.:

1967 Elastic waves in layered media, Geophys. Prosp., 15, 4, 644-650.

BREKHOVSKIKH, L, M.:

1960 Waves in layered media, Transl. by D. Liebermann, Acad. Press, New York, 561p.

ČERVENÝ, V.:

1961 The amplitude curves of reflected harmonic waves around the critical point, Studia geoph. et geod., 5, 4, 319-351.

COOLEY, J. W. and J. W. TuKeY:

1965 An algorithm for the machine calculation of complex Fourier series, Math. of Computation, 19, 90, 297-301.

DATTA, B.:

1967 Das Spektrum seismischer Wellen bei der Reflexion an einer Übergangsschicht, Ztschr. f. Geophys., 33, 2, 80-93.

FUCHS, K.:

1968 Die Reflexion von Kugelwellen an inhomogenen Übergangszonen mit beliebiger Tiefenverteilung der elastischen Moduln und der Dichte, Habilitation Thesis, University Karlsruhe, 136p.

FUCHS, K.:

1969a The method of stationary phase as a diagnostic aid in estimating the field pattern of body waves reflected from transition zones, Ztschr. f. Geophys., 35, (in press).

FúCHS, K.:

1969b The method of stationary phase applied to the reflection of spherical waves from transition zones with arbitrary depthdependent elastic moduli and density, Ztschr. f. Geophys., 35, (in press).

GRANT, F.S. and G.F. WeSt:

1965 Interpretation theory in applied geophysics, McGraw-Hill, New York, 538p.

GUPTA, R. N.:

1966 Reflection of elastic waves from a linear transition layer, Bull. Seism. Soc. Amer., 56, 2, 511-526.

HARKRIDER, D. G.:

1964 Surface waves in multilayered elastic media. I. Rayleigh and Love waves from buried sources in a multilayered elastic half-space, Bull. Seism. Soc. Amer., 54, $2,627-679$.

HASKELL, N.A.:

- 1953 The dispersion of surface waves in multi- layered media, Bull. Seism. Soc. Amer., 43, 17-34.

HEELAN, P. A.:

1953 On the theory of head waves, Geophysics, 18, 871-893.

HELMBERGER, D. V.:

1968 The crust mantle transition in the Bering Sea, Bull. Seism. Soc. Amer., 58, 1, 179214.

Hook, J. F.:

1961 Separation of vector wave equation of elasticity for certain types of isotropic, inhomogeneous media, Journ. Acoust. Soc. Amer., 33, 302-313.

Hook, J. F.:

1962a Generalization of a method of potentials for the vector wave equation of elasticity for inhomogeneous media, Journ. Acoust. Soc. Amer., 34, 354-355.

Hook, J. F.:

1962b Contributions to a theory of separability of the vector wave equation of elasticity for inhomogeneous media, Journ. Acoust. Soc. Amer., 34, 946-953.

Hook, J. F.:

1965 Determination of inhomogeneous media for which the vector wave equation of elasticity is separable, Bull. Seism. Soc. Amer., 55, 975-987.

MENZEL, $H$. and O. ROSENBACH:

1957. Theoretische Untersuchungen über den Einfluß der Verwitterungsschicht auf das Spektrum elastischer Wellen in der Reflexionsseismik, Geophys. Prosp., 5, 3, 328-348.

MENZEL, H. and O. ROSENBACH:

1958a The influence of a layer complying with a linear velocity law on the shape of seismic pulses, Geophys. Prosp., 6, 408432.

MENZEL, H. and O. ROSENBaCH:

1958b Bemerkungen über den Einfluß der Verwitterungsschicht auf das Spektrum seismischer Wellen, Geofis. Pura e Appl., 40, 36-40.

MÜLLER, G.:

1964 Elastische Kugelwellen und ihre Reflexion und Brechung an der ebenen Trennfläche zwischen zwei homogenen, isotropen Halbräumen, Diplomarbeit, Universität Mainz.

MÜLLER, G.:

1967 Theoretische Seismogramme für Punkt- 
quellen in geschichteten Medien, Dissertation, Technische Hochschule Clausthal, 136p.

PERERIS, C. L.:

1955a The seismic surface pulse, Proc. Nat. Acad. Sci. U.S., 41, 469-480.

PEKERIS, C. L.:

1955b The seismic buried pulse, Proc. Nat. Acad. Sci. U.S., 41, 629-639.

PEKERIS, C.L.:

1960 Propagation of seismic pulses in layered liquids and solids, Internat. Sympos. on Stress Wave Propagation in Materials (ed. N. Davids), Interscience Publishers, New York, 45-57.

PEKERIS, C. L. and H. LIFSON:

1957 Motion of the surface of a uniform halfspace produced by a buried pulse, Journ. Acoust. Soc. Amer., 29, 1233-1238.

Pekeris, C. L., Z. Alterman and F, Abramovici:

1963 Propagation of an SH-torque pulse in a layered solid, Bull. Seism. Soc. Amer., 53, $39-57$.

Pekeris, C. L., Z. Alterman, F. Abramovici and H. JAROSCH:

1965. Propagation of a compressional pulse in a layered solid, Rev. Geophys., 3, 25-47.
PhinNey, R. A.:

1965 Theoretical calculations of the spectrum of first arrivals in layered elastic mediums, Journ, Geophys, Res., 70, 5107-5123.

SCHOLTE, J. G. J.:

1961 Propagation of waves in inhomogeneous media, Geophys. Prosp., 9, 86-115.

SEZAWA, K. and K. KANAI:

1935 The effect of sharpness of discontinuities on the transmission and reflection of elastic waves, Bull. Earthq. Res. Inst. Tokyo Univ., 13, 750-756.

SPENCER, T. W.:

1965 Long-time response predicted by exact elastic ray theory, Geophysics, 30, 363-368.

SPENCER, T.W.:

1965 Refraction along a layer, Geophysics, 30, 3, 369-388.

THOMSON, W.T.:

1950 Transmission of elastic waves through a stratified medium, Journ. Appl. Phys., 21, 86-93.

WoLF, A.:

1937 The reflection of elastic waves from transition layers of variable velocity, Geophysics, 2, 357-363.

(Received Nov. 27, 1968) 